\title{
Surgical treatment of postintubation tracheal
} stenosis: Iranian experience of effect of previous tracheostomy

This article was published in the following Dove Press journal:

International Journal of General Medicine

24 January 2012

Number of times this article has been viewed

\section{Shahryar Hashemzadeh' \\ Khosrow Hashemzadeh ${ }^{2}$ \\ Farzad Kakaei ${ }^{3}$ \\ Raheleh Aligholipour ${ }^{4}$ \\ Kamyar Ghabili ${ }^{5}$}

'Tuberculosis and Lung Disease Research Center, ${ }^{2}$ Department of Cardiovascular Surgery, Shahid Madani Hospital, ${ }^{3}$ Department of General Surgery, ${ }^{4}$ Students Research Committee, ${ }^{5}$ Medical Philosophy and History Research Center, Tabriz University of Medical Sciences, Tabriz, Iran
Correspondence: Kamyar Ghabili Medical Philosophy and History Research Center, Tabriz University of Medical Sciences, Tabriz, Iran Tel +989144106136

Fax +98 4I 13292944 Email kghabili@gmail.com
Background: Postintubation tracheal stenosis remains the most common indication for tracheal surgery. In the event of a rapid and progressive course of the disease after extubation, surgical approaches such as primary resection and anastomosis or various methods of tracheoplasty should be selected. We report our experience with surgical management of moderate to severe postintubation tracheal stenosis. We also compared intraoperative variables in postintubation tracheal stenosis between those with and without previous tracheostomy.

Methods: Over a 5-year period from June 2005 to July 2010, 50 patients aged 14-64 years with moderate $(50 \%-70 \%$ of the lumen) to severe $(>70 \%)$ postintubation tracheal stenosis underwent resection and primary anastomosis. Patients were followed up to assess the surgical outcome. To study the effect of previous tracheostomy on treatment, surgical variables were compared between patients with previous tracheostomy (group A, $\mathrm{n}=27$ ) and those without previous tracheostomy (group B, $\mathrm{n}=23$ ).

Results: Resection and primary anastomosis was performed via either cervical incision (45 patients) or right thoracotomy (five patients). In two patients with subglottic stenosis, complete resection of the tracheal lesion and anterior portion of cricoid cartilage was performed, and the remaining trachea was anastomosed to the thyroid cartilage using a Montgomery T-tube. There was only one perioperative death in a patient with a tracheo-innominate fistula. The length of the resected segment, number of resected rings, and subsequent duration of surgery were significantly greater in group A compared with group B $(P<0.05)$. Six months after surgery, the outcome was satisfactory to excellent in 47 (95.9\%) patients.

Conclusion: This surgical approach leads to highly successful results in the treatment of moderate to severe postintubation tracheal stenosis. In addition, previous tracheostomy might prolong the duration of surgery and increase the need for postoperative interventions due to an increase in the length and number of resected tracheal segments. Therefore, in the event of emergency tracheostomy in postintubation tracheal stenosis, insertion of the tracheostomy tube close to the stenotic segment is recommended.

Keywords: trachea, tracheostomy, tracheal stenosis, intubation, tracheal resection

\section{Introduction}

Postintubation tracheal stenosis is a clinical problem caused by pressure necrosis of the airway. The incidence of postintubation tracheal stenosis has decreased with recognition of its etiology and modifications in the design and management of endotracheal and tracheostomy tubes; however, it remains the most common indication for tracheal surgery. ${ }^{1}$ In a patient with a recent tracheal stenosis, the airway can be managed initially with rigid bronchoscopic dilation or other treatment modalities such as stenting, percutaneous dilatation, fiberoptic-assisted balloon dilatation, argon plasma coagulation, and laser 
therapy with or without stenting. ${ }^{2}$ The major determinant of the treatment method used is whether postintubation damage extends to the tracheal cartilage. Even in successful cases of nonsurgical management, stenosis will eventually recur due to the extent of necrosis. Therefore, surgical treatment would be the only definitive treatment modality in such cases. $^{3}$ For a patient with a rapid and progressive course of the disease after extubation, surgical approaches such as primary resection and anastomosis or various methods of tracheoplasty should be selected. ${ }^{4}$ Emergency tracheostomy is a management strategy for patients in very poor condition or in centers where surgical expertise is lacking, resulting in further complications during subsequent definitive surgical repair. We report our experience of the surgical management of moderate to severe postintubation tracheal stenosis in a consecutive series of 50 patients treated in the previous 5 years. Moreover, we compared intraoperative variables in postintubation tracheal stenosis between those with and without previous tracheostomy.

\section{Methods}

\section{Patients and study protocol}

Over a 5-year period from June 2005 to July 2010, 50 patients aged $14-64$ years with moderate $(50 \%-70 \%$ of the lumen) to severe $(>70 \%)$ postintubation tracheal stenosis were admitted to our center for surgical repair. In all of the patients studied, tracheal stenosis was a complication of their hospital course in the intensive care unit. Patients with mild tracheal stenosis ( $<50 \%$ of the lumen) treated with bronchoscopic dilatation and those who remained asymptomatic after bronchoscopic dilatation for over 6 months were excluded. Initial preoperative diagnostic evaluation included pulmonary function tests, computed tomography, and fiberoptic and rigid bronchoscopic examinations. Resection and primary anastomosis was performed via either a cervical incision or right thoracotomy. Preoperative bronchoscopic dilatation was performed where indicated. Patients were followed up to assess the surgical outcome. To study the effect of previous tracheostomy on treatment, surgical variables including length of resected segment, number of resected rings, duration of surgery, total intensive care unit stay and duration of hospitalization were compared between patients with previous tracheostomy (group $A, n=27$ ) and without previous tracheostomy (group $\mathrm{B}, \mathrm{n}=23$ ).

\section{Surgical approach}

All surgical procedures were performed under general anesthesia using standard techniques for airway surgery.
A rigid bronchoscope was used at the time of induction for intubation. After the stenotic segment was resected, ventilation was performed through a spiral tracheal tube inserted in the distal tracheal segment. In patients with previous tracheostomy, existing stomas were used for intubation. To enable safe anastomosis, complete resection of the stenotic lesions was performed between proximal and distal disease-free cartilage. Careful mucomucosal approximation at the anastomosis was done using interrupted 4-0 polyglactin (Vicryl) absorbable sutures, with knots tied outside the lumen. In patients with previous tracheostomy, the stoma site as well as the stenotic lesion was resected. In patients with subglottic stenosis, complete resection of the tracheal lesion and anterior portion of the cricoid cartilage was performed, and the remaining trachea was anastomosed to the thyroid cartilage. In those with intact cricoid cartilage, tracheal resection and cricotracheal anastomosis was performed. Besides the tracheal resection, suprahyoid laryngeal release, pericardial incision and mobilization of the right lung hilus, and laryngeal release and hilus mobilization were used to reduce tension on the anastomosis. ${ }^{5}$ All anastomoses in these patients were done using interrupted 4-0 Vicryl absorbable sutures, with the knots tied outside the lumen.

\section{Postoperative care}

At the end of the procedure, the patient's chin was sutured with heavy silk to the presternal skin in neck flexion to prevent sudden hyperextension or tension on the anastomosis. These sutures were removed after one week and prior to hospital discharge. All patients were extubated in the operating room. The patients were given low-dose hydrocortisone and prophylactic intravenous antibiotics for the first 24 hours after surgery.

\section{Outcome}

The outcome was classified as excellent, good, satisfactory, or not satisfactory according to patient status at the time of discharge and after 6 months. ${ }^{3}$ The results were classified as "excellent" if voice and respiration were completely normal. "Good" results were judged in the presence of slight lessening of maximum voice volume, slight hoarseness, slight weakness of the voice after prolonged use, and adequate breathing for normal activities. Results were classified as "satisfactory" in the case of a hoarse voice with slight wheezing or shortness of breath on exercise, not sufficient to impair normal activities. Results were "not satisfactory" in the event of major complications and need for numerous postoperative bronchoscopic dilatations. 


\section{Statistical analysis}

Data are presented as the mean \pm standard deviation or percentage. Statistical analysis was performed with SPSS for Windows (v 13.0; SPSS Inc, Chicago, IL) using the Chi-squared test, Fisher's Exact test, or independent-samples $t$-test, wherever appropriate. A $P$ value $<0.05$ was considered to be statistically significant.

\section{Results}

Fifty patients, comprising 32 (64\%) men and 18 (36\%) women, were recruited for the present study. The severity of tracheal stenosis was moderate in five patients and severe in 45 patients, respectively. Thirty-nine patients had a history of bronchoscopic dilatation 1-5 times before operation but stenosis recurred in these patients after 1-5 months. Emergency preoperative bronchoscopic dilatation was indicated in six patients during the same hospital admission before definitive repair. Patients with previous tracheostomy $(\mathrm{n}=27)$ had prolonged intubation $(\mathrm{n}=10)$ or previous postintubation tracheal stenosis $(\mathrm{n}=17)$. The latter group underwent tracheostomy in other centers due to lack of expertise $(n=12)$, bad general condition, or inflammatory status of the trachea $(n=5)$. Table 1 shows the patients' preoperative variables, including age, causal factors for prolonged intubation, location of stenosis, duration of the intubation, and time interval between extubation/ decannulation and onset of symptoms.

Resection and primary anastomosis was performed via a cervical incision in 45 patients. Right thoracotomy was required in five patients who had thoracic $(n=3)$ and supracarinal $(\mathrm{n}=2)$ stenosis. Partial sternal split was added to the cervical incision in three patients with cervicothoracic tracheal stenosis. In two patients with subglottic stenosis, complete resection of the tracheal lesion and anterior portion of cricoid cartilage was performed and the remaining trachea was anastomosed to the thyroid cartilage using a Montgomery T-tube. The T-tube was removed without any complication 3 months later without any anesthesia. In the other four patients with subglottic stenosis and intact cricoid cartilage, tracheal resection and cricotracheal anastomosis was performed.

Mean length of the resected segment was $3.66 \pm 1.01$ (range 2-6) $\mathrm{cm}$. The mean number of the resected rings was $5.46 \pm 1.43$ (range 3-10). Mean duration of surgery, intensive care unit stay, and total hospitalization was $3.61 \pm 0.64$ hours, $3.42 \pm 1.52$ days, and $9.30 \pm 2.28$ days, respectively. Length of the resected segment as well as number of resected rings was significantly greater in group A compared with group B $(P<0.05$, Table 2$)$. Subsequently, the duration of surgery was significantly prolonged in group A compared with group B (4.05 \pm 0.44 hours versus $3.10 \pm 0.41$ hours, $P=0.0001$, Table 2).

Minor perioperative complications including superficial wound infection (four patients), temporary vocal cord dysfunction (four patients), and pneumonia (two patients) occurred in nine patients. We had only one inhospital death. This patient with a history of previous brain tumor surgery developed postoperative mediastinitis and severe bleeding secondary to a tracheo-innominate fistula. At the time of hospital discharge, the outcome was excellent in 33 (67.3\%), good in $11(22.4 \%)$, and satisfactory in five (10.2\%) patients (Table 3).

Table I Preoperative variables according to tracheostomy status

\begin{tabular}{|c|c|c|c|}
\hline Variable & $\begin{array}{l}\text { Tracheostomy } \\
(\mathrm{n}=27)\end{array}$ & $\begin{array}{l}\text { No tracheostomy } \\
(n=23)\end{array}$ & $P$ value \\
\hline Age & $32.92 \pm 15.95$ & $34.21 \pm 13.89$ & 0.75 \\
\hline Cause of prolonged intubation & $21(77.8 \%)$ & $10(43.5 \%)$ & NA \\
\hline Multiple trauma & I (3.7\%) & 7 (34.4\%) & \\
\hline Suicide attempts & $0(0 \%)$ & $6(26.1 \%)$ & \\
\hline Cerebral vascular accident & $3(11.1 \%)$ & $0(0 \%)$ & \\
\hline Chronic pulmonary disease & $2(7.4 \%)$ & $0(0 \%)$ & \\
\hline Location of stenosis & & & NA \\
\hline Subglottic & $3(11.1 \%)$ & $3(13 \%)$ & \\
\hline Cervical & $21(77.8 \%)$ & $16(69.6 \%)$ & \\
\hline Cervicothoracic & $2(7.4 \%)$ & I (4.3\%) & \\
\hline Thoracic & I (3.7\%) & $2(8.7 \%)$ & \\
\hline Supracarinal & $0(0 \%)$ & I (4.3\%) & \\
\hline Duration of intubation (days) & $9.00 \pm 3.62$ & $11.07 \pm 4.10$ & 0.06 \\
\hline $\begin{array}{l}\text { Time interval between extubation } \\
\text { and stenosis (days) }\end{array}$ & $23.13 \pm 22.23$ & $27.62 \pm 20.55$ & 0.44 \\
\hline
\end{tabular}

Abbreviation: NA, not available. 
Table 2 Intraoperative variables according to preoperative tracheostomy status

\begin{tabular}{llll}
\hline Variable & $\begin{array}{l}\text { Tracheostomy } \\
(\mathbf{n}=\mathbf{2 7})\end{array}$ & $\begin{array}{l}\text { No tracheostomy } \\
(\mathbf{n}=\mathbf{2 3})\end{array}$ & $\begin{array}{c}\text { P value } \\
\text { Length of resected segment }(\mathrm{cm})\end{array}$ \\
Number of resected rings & $4.13 \pm 0.95$ & $3.11 \pm 0.78$ & 0.000 \\
Duration of surgery (hours) & $6.04 \pm 1.60$ & $4.78 \pm 0.79$ & 0.001 \\
Total intensive care stay (days) & $4.05 \pm 0.44$ & $3.10 \pm 0.41$ & 0.000 \\
Total hospitalization (days) & $3.37 \pm 1.50$ & $3.48 \pm 1.59$ & 0.807 \\
\hline
\end{tabular}

The patients were followed up from 6 to 43 (median 17) months. Six months after the operation, the outcome was excellent in $31(63.3 \%)$, good in $13(26.5 \%)$, and satisfactory in three $(6.1 \%)$ patients. Meanwhile, the surgical outcome was not satisfactory in two $(4.1 \%)$ patients due to the need for numerous bronchoscopic dilatations. These two patients had subglottic tracheal stenosis secondary to the previous tracheostomy. Table 3 shows the values of the surgical outcome in groups A and B and in total.

\section{Discussion}

The present study revealed an acceptable surgical outcome (95.9\% excellent to satisfactory results) in a group of patients with postintubation tracheal stenosis. This finding is consistent with previous research. ${ }^{3,6-8}$ In contrast, Abbasidezfouli et al achieved a $61.5 \%$ success rate in surgical treatment of the postintubation multisegmental tracheal stenosis. ${ }^{9}$ This discrepancy might be attributed to the complexity of therapeutic approaches toward multisegmental tracheal stenosis. Moreover, Grillo et $\mathrm{al}^{7}$ reported a failure rate similar to that of the present study (about 4\%). However, Rea et al had no "not satisfactory" results in their surgical treatment of benign tracheal and laryngotracheal stenosis. ${ }^{3}$ Although their study included patients with postintubation tracheal stenosis as well, no individuals had a previous history of tracheostomy. ${ }^{3}$ This feature might have accounted for their lack of cases of surgical failure. In the present study, both cases of "not satisfactory" results had subglottic stenosis due to previous tracheostomy. Furthermore, the perioperative mortality rate in our study was quite similar to that of previous studies. ${ }^{3,7,8}$

The present study also demonstrates that previous tracheostomy significantly increases the duration of surgery, length of tracheal resection, and number of resected rings, but no significant effect on intensive care unit stay or total hospitalization. To the best of our knowledge, the present study is the first to compare intraoperative variables in postintubation tracheal stenosis between patients with and without previous tracheostomy. In those who previously underwent tracheostomy for transient relief of respiratory symptoms of postintubation tracheal stenosis, we had to sacrifice the normal trachea between the tracheostomy stoma and stenosis site, and this increased the length of resection and duration of surgery. This would theoretically increase the tension on

Table 3 Outcome of operation at time of hospital discharge and 6 months after surgery*

\begin{tabular}{llll}
\hline Outcomes & $\begin{array}{l}\text { Tracheostomy } \\
(\mathbf{n}=\mathbf{2 7})\end{array}$ & $\begin{array}{l}\text { No tracheostomy } \\
(\mathbf{n}=\mathbf{2 2})\end{array}$ & $\begin{array}{l}\text { Total } \\
(\mathbf{n}=\mathbf{4 9})\end{array}$ \\
\hline Outcome at hospital discharge & & & \\
Excellent & $17(63 \%)$ & $16(72.7 \%)$ & $33(67.3 \%)$ \\
Good & $7(25.9 \%)$ & $4(18.2 \%)$ & $11(22.4 \%)$ \\
Satisfactory & $3(11.1 \%)$ & $2(9 \%)$ & $5(10.2 \%)$ \\
Not satisfactory & $0(0 \%)$ & $0(0 \%)$ & $0(0 \%)$ \\
Outcome 6 months after operation & & & $31(63.3 \%)$ \\
Excellent & $15(55.6 \%)$ & $16(72.7 \%)$ & $13(26.5 \%)$ \\
Good & $8(29.6 \%)$ & $5(22.7 \%)$ & $3(6.1 \%)$ \\
Satisfactory & $3(11.1 \%)$ & $0(0 \%)$ & $2(4.1 \%)$ \\
Not satisfactory & $1(3.7 \%)$ & $1(4.5 \%)$ & $9(18.4 \%)$ \\
Minor complications & $5(18.5 \%)$ & $4(18.2 \%)$ & $4(8.2 \%)$ \\
Bronchoscopic dilatation & & & $5(10.2 \%)$ \\
I & $3(11.1 \%)$ & $1(4.5 \%)$ & $2(4.1 \%)$ \\
2 & $4(14.8 \%)$ & $1(4.5 \%)$ & 0 \\
$\geq 3$ & $2(7.4 \%)$ & $0(0 \%)$ & \\
\hline Not & & & \\
\hline
\end{tabular}

Note: *One mortality case was excluded from the outcome assessment. 
the anastomosis and the possibility of restenosis. In practice, the need for postoperative bronchoscopic dilatation was greater in patients with previous tracheostomy in the present study. In other words, tracheostomy itself may be considered as a factor of increased length of resection and subsequent restenosis.

Despite technological improvements and more skillful patient care in the intensive care unit, tracheal and laryngotracheal stenoses still constitute an important group of iatrogenic sequelae after intubation and tracheostomy. ${ }^{6,10}$ The reported incidence of tracheal stenosis following tracheostomy and laryngotracheal intubation ranges from $0.6 \%$ to $21 \%$ and $6 \%$ to $21 \%$, respectively. ${ }^{6}$ Studies have shown that the main cause is pressure exerted on the tracheal mucosa by the cuff. Any pressure over $30 \mathrm{mmHg}$ exceeding the mucosal capillary perfusion pressure causes mucosal ischemia and leads to ulceration and subsequent stenosis. ${ }^{11}$ Using a tracheostomy tube with a high volume and low pressure cuff has been shown to reduce postintubation injury. However, prolonged ventilatory support with tracheostomy still affects the trachea despite best nursing care. ${ }^{12}$ Damaging the cartilage during tracheostomy or wound sepsis at the stoma site with subsequent granulation tissue formation are other causes of tracheal stenosis in those patients who need tracheostomy for airway protection. ${ }^{6}$

The current treatment strategies for tracheal stenosis vary widely. Apart from its preoperative application for diagnosis of tracheal stenosis, rigid bronchoscopy plays an important role in maintenance of a safe airway prior to the surgical treatment and, in some cases, definitive management of stenosis through dilatation. ${ }^{1,3,13}$ Alternatively, laser therapy can be applied to cicatrized lesions leading to airway stenosis. However, definitive management of only thin webline strictures and damage to the cricoid posterior plate in repeated applications has limited the use of laser in treatment of tracheal stenosis. ${ }^{3,14}$ Laser therapy is now recommended only in patients with true contraindications to surgery. ${ }^{3}$ Currently, the best treatment for postintubation tracheal stenosis is a surgical approach accompanied by the basic principles of tracheal reconstruction, including appropriate reduction of tension, maintenance of tracheal blood supply, and meticulous dissection and anastomosis. ${ }^{1}$ Nonetheless, treatment of extensive lesions remains an unsolved problem. Therefore, recent studies have been initiated to investigate plausible treatment alternatives (eg, a tissue-engineered airway, revascularized allografts in the heterotopic position, and cryopreserved aortic allografts) for extensive lesions in postintubation tracheal stenosis. ${ }^{15-17}$
In conclusion, our findings confirm that a surgical approach leads to highly successful results in the treatment of moderate to severe postintubation tracheal stenosis. In addition, previous tracheostomy might prolong duration of surgery and increase the need for postoperative interventions due to an increase in length and number of resected tracheal segments. Therefore, in the event of emergency tracheostomy in postintubation tracheal stenosis, insertion of a tracheostomy tube close to the stenotic segment is recommended. Moreover, treatment of subglottic stenosis might require use of a Montgomery T-tube to support the anastomosis.

\section{Disclosure}

The authors report no conflicts of interest in this work.

\section{References}

1. Wain JC Jr. Postintubation tracheal stenosis. Semin Thorac Cardiovasc Surg. 2009;21:284-289.

2. Softah A. Rigid bronchoscopic dilatation of postintubation tracheal stenosis. West Afr J Med. 2005;24:234-238.

3. Rea F, Callegaro D, Loy M, et al. Benign tracheal and laryngotracheal stenosis: surgical treatment and results. Eur J Cardiothorac Surg. 2002;22:352-356.

4. Grillo HC. Postintubation stenosis. In: Grillo HC, editor. Surgery of the Trachea and Bronchi. Hamilton, Canada: BC Decker Inc; 2004.

5. Hashemzade S, TaghizadiehA, Rashidi F, Esmaeili HA, Mohammadreza G. Mucoepidermoid carcinoma of trachea in a 22 year old woman: a case report. Pneumologia. 2011;60:164-165.

6. Sarper A, Ayten A, Eser I, et al. Tracheal stenosis after tracheostomy or intubation: review with special regard to cause and management. Tex Heart Inst J. 2005;32:154-158.

7. Grillo HC, Donahue DM, Mathisen DJ, et al. Postintubation tracheal stenosis. Treatment and results. $J$ Thorac Cardiovasc Surg. 1995;109:486-492.

8. Wright CD, Grillo HC, Wain JC, et al. Anastomotic complications after tracheal resection: prognostic factors and management. J Thorac Cardiovasc Surg. 2004;128:731-739.

9. Abbasidezfouli A, Shadmehr MB, Arab M, et al. Postintubation multisegmental tracheal stenosis: treatment and results. Ann Thorac Surg. 2007;84:211-214.

10. Couraud L, Jougon JB, Velly JF. Surgical treatment of nontumoral stenoses of the upper airway. Ann Thorac Surg. 1995;60:250-259.

11. Spittle N, McCluskey A. Lesson of the week: tracheal stenosis after intubation. BMJ. 2000;321:1000-1002.

12. Tsubota N, Yoshimura M, Miyamoto Y, et al. Primary anastomosis of the trachea: management and pitfalls. Surg Today. 1998;28:492-497.

13. Carretta A, Melloni G, Ciriaco P, et al. Preoperative assessment in patients with postintubation tracheal stenosis: Rigid and flexible bronchoscopy versus spiral CT scan with multiplanar reconstructions. Surg Endosc. 2006;20:905-908.

14. Sharpe DAC, Dixon K, Moghissi K. Endoscopic laser treatment for tracheal obstruction. Eur J Cardiothorac Surg. 1996;10:722-726.

15. Macchiarini P, Jungebluth $P, G o T$, et al. Clinical transplantation of a tissue-engineered airway. Lancet. 2008;372:2023-2030.

16. Delaere P, Vranckx J, Verleden G, De Leyn P, Van Raemdonck D; Leuven Tracheal Transplant Group. Tracheal allotransplantation after withdrawal of immunosuppressive therapy. $N$ Engl $J$ Med. 2010;362:138-145.

17. Martinod E, Radu DM, Chouahnia K, et al. Human transplantation of a biologic airway substitute in conservative lung cancer surgery. Ann Thorac Surg. 2011;91:837-842. 


\section{Publish your work in this journal}

The International Journal of General Medicine is an international, peer-reviewed open-access journal that focuses on general and internal medicine, pathogenesis, epidemiology, diagnosis, monitoring and treatment protocols. The journal is characterized by the rapid reporting of reviews, original research and clinical studies across all disease areas.

A key focus is the elucidation of disease processes and management protocols resulting in improved outcomes for the patient. The manuscript management system is completely online and includes a very quick and fair peer-review system. Visit http://www.dovepress.com/ testimonials.php to read real quotes from published authors.

Submit your manuscript here: http://www.dovepress.com/international-journal-of-general-medicine-journal 